\title{
La aplicación práctica de la farmacoeconomía en la utilización de los medicamentos en Cuba
}

\author{
Manuel M. Collazo Herrera*
}

Centro de Investigación y Desarrollo de Medicamentos (CIDEM), Ciudad de La Habana, Cuba

${ }^{*}$ Correspondencia:

M. C. Herrera

Dirección: Calle Cuarta Nro. 119/J.

Martí y Mella, Rpto. Eléctrico

Arroyo Naranjo

CP 10900

Ciudad de La Habana - Cuba

Email: cidem@infomed.sld.cu
El objetivo de este trabajo es exponer cómo los estudios farmacoeconómicos contribuyen a darle un uso más racional y eficiente a los medicamentos. Como consecuencia del enfoque actual que le dan las autoridades sanitarias de la República de Cuba a la farmacoeconomía, la industria farmacéutica se ha empezado a interesar por dichas técnicas analíticas y a utilizarlas para desarrollar sus estrategias de investigación-desarrollo, producción y comercialización de sus productos. La aplicación práctica de estas técnicas de análisis al campo sanitario, trae como resultado una implementación eficiente del tratamiento farmacológico para la obtención de un mayor beneficio en términos de salud del paciente, a un costo más razonable para la economía nacional. Como conclusión de este análisis, se exponen algunos ejemplos prácticos de estudios farmacoeconómicos realizados de conjunto entre el sector farmacéutico y el de asistencia médica, referidos a los tratamientos farmacológicos antiulcerosos y a la profilaxis perioperatoria de antimicrobianos, demostrando asi su aplicación en la toma de decisiones sanitarias, sobre la base de la efectividad clínica comprobada y los costos de la farmacoterapéutica empleada.

\section{INTRODUCCION}

La aplicación de los conocimientos y técnicas de la evaluación económica al campo de los medicamentos permite centrar el análisis en el tratamiento de los problemas que plantea una adecuada prescripción, tanto desde el punto de vista de la efectividad, como de la eficiencia; es decir, de la obtención de un beneficio social en términos de salud, a un costo razonable (Glennie, 1995; Trillo, Aznar, 1999).
Unitermos

- Farmacoeconomía

- Costo-efectividad

- Tratamientos antiulcerosos

- Profilaxis post-operatoria

- Uso racional 
en Cuba, se le otorga una especial importancia a la farmacoeconomía.

Como demostración de su aplicación práctica, se exponen algunas investigaciones realizadas en los distintos niveles de la atención de salud, una de ellas referente a los tratamientos farmacológicos antiulcerosos, comparando la utilización de los esquemas de ranitidina y del omeprazol, y otro estudio con respecto a la quimioprofilaxis perioperatoria de antimicrobianos, evaluando las opciones alternativas con la administración de cefalosporinas, como es el caso del empleo de cefazolina y ceftriaxona en la prevención de sepsis post-quirúrgicas.

\section{La importancia de la farmacoeconomía para la eficiencia y el uso racional de los medicamentos}

La evaluación económica de medicamentos se engloba dentro de una disciplina más amplia: la evaluación económica de tecnologías sanitarias. El fin último de este tipo de evaluaciones es la selección de aquellas opciones que tengan un impacto sanitario más positivo (Sacristán del Castillo, 1995).

Puesto que toda evaluación se basa en la comparación, la evaluación económica de medicamentos podría definirse como la determinación de la eficiencia (relación entre costos y efectos) de un tratamiento farmacológico y su comparación con la de otras opciones, con el fin de seleccionar aquella con una relación costo/efecto más favorable (Sacristán del Castillo, 1995).

Desde una perspectiva clínica, la utilización de una tecnología se justifica si su efectividad (la consecución de un objetivo en condiciones reales) o, por lo menos, su eficacia (la consecución de un objetivo en condiciones ideales) es positiva. Sin embargo, desde la perspectiva económica el acento se pone en la eficiencia (Badia, Rovira, 1994). Por lo tanto, la evaluación económica consiste en determinar los efectos que se derivarían de seguir cada una de las opciones o subsiguientes cursos de acciones posibles en una situación de elección y compararlas, en términos de su eficiencia social, es decir, de su contribución a la maximización del bienestar de la sociedad (Drummond, Stoddart, Torrance, 1987)

La evaluación económica de tecnología y programas sanitarios y, especialmente de productos farmacéuticos, se está utilizando cada vez con mayor frecuencia para hacer frente al hecho de que los recursos deben utilizarse con la mayor eficiencia posible para lograr el máximo beneficio de los pacientes (Panerai, Mohr, 1990). La industria farmacéutica se está involucrando cada vez más en la evaluación económica de los medicamentos que desarrolla, ya que las aplicaciones prácticas de los estudios farmacoeco- nómicos pueden ser útiles en multitud de situaciones relacionadas con la toma de decisiones (Guía directiva para la evaluación económica de medicamentos: Canadá, 1994).

En la actualidad, y dado el elevado costo que supone el desarrollo de un nuevo producto, existe dentro de las compañías farmacéuticas una clara vocación y orientación en proporcionar fármacos cada vez más eficientes, a la vez más eficaces, mejor tolerados y de mayor calidad, lo que supondrá un claro avance y mejora dentro del arsenal terapéutico disponible (Soto, 1999). Por este motivo, dentro de la industria farmacéutica cada vez se están diseñando y realizando análisis de evaluación económica de medicamentos con más asiduidad durante todo su ciclo de vida, tanto durante los primeros estadios de su desarrollo como una vez hayan sido comercializados (Anderson, 1995). Esto hecho refleja la necesidad de prioridad de inversión de los recursos existentes y escoger los medicamentos que presente una mejor relación costo-efectividad (o sea que sean más eficientes), y que por lo tanto logren el mayor beneficio terapéutico con el menor costo asociado posible (Sacristán del Castillo et al., 1994).

En sociedades industrializadas capaces de regular y controlar los costos de producción de bienes de salud y donde la cobertura sanitaria es prácticamente total, la farmacoeconomía podrá sin duda ayudar a mejorar la toma de decisiones clínicas, sobre todo cuando se debe escoger entre varios tratamientos similares. Todo ejercicio, sin embargo, debe estar enfocado en la perspectiva, no-solo de controlar costos sino de mejorar la eficiencia (Evans, 1992) para alcanzar la equidad en el acceso a los servicios de salud.

En los países en desarrollo, donde una parte importante de la población no tiene acceso a medicamentos y donde muchas personas mueren a causa de enfermedades para las cuales existen medicamentos preventivos, la farmacoeconomía tendrá un enfoque y unas características muy diferentes. Más que contención de costos, se buscará racionalizar los recursos existentes y conseguir fuentes suplementarias de financiamiento, de tal forma que garantice la extensión de la cobertura a toda la población (Velázquez, 1999).

Para estos países, la evaluación económica del medicamento debe enmarcarse en un análisis macroeconómico que aborde el problema de los recursos disponibles, con frecuencia limitados, en relación con las necesidades globales. Una farmacoeconomía de este tipo estaría muchísimo más centrada en la selección de fármacos, y la evaluación económica del medicamento sería también un elemento más para promover lo que la OMS ha llamado, el uso racional del medicamento (Velázquez, 1999).

Debido a que Cuba, aun cuando es un país del Ter- 
cer Mundo, cuenta con un sistema sanitario comparable a los que tienen los países más desarrollados, la farmacoeconomía en esta nación tendrá un doble propósito: racionalizar el uso de los medicamentos seleccionados y mejorar los niveles de eficiencia de los tratamientos (Collazo, Casademunt, 2001).

Es por esta razón, que se han realizado los primeros estudios farmacoeconómicos por parte del Centro de Investigación y Desarrollo de Medicamentos (CIDEM), perteneciente al Ministerio de Salud Pública de la República de Cuba; para darle una mejor utilización a los medicamentos con relación a los problemas de salud que tiene la población, dados los limitados recursos económicos disponibles con que cuenta el país.

\section{Estudios de evaluación económica de fármacos realizados en Cuba}

En Cuba se han realizados diferentes estudios farmacoeconómicos con el objetivo de elaborar y poner en práctica al nivel nacional, una política para el uso racional de los medicamentos que esté acorde a la situación económico-financiera que confronta el país; así como de elevar los niveles de eficiencia de los tratamientos farmacológicos para el Sistema Nacional de Salud.

Los ejemplos de casos prácticos que se exponen, corresponden a tipos de estudios de análisis de costo-efectividad referentes a dos problemas de salud que confronta nuestro sistema sanitario nacional, como son:

- Cicatrización de las úlceras pépticas y erradicación de agente causante (Helicobacter pylori).

- Prevención de las infecciones post-operatorias intrahospitalarias con el uso de antimicrobianos.

\section{EVALUACIÓN ECONÓMICA DE LOS TRATAMIENTOS FARMACOLÓGICOS ANTIULCEROSOS}

\section{Materiales Y Metodos}

En el caso del estudio sobre la curación de las úlceras, se evaluaron dos alternativas de tratamientos farmacológicos conformados por diferentes medicamentos, todas estas opciones con un ciclo de duración de cuatro semanas. Para este objetivo se emplea un esquema quimioterapéutico de terapia triple convencional, que contienen un agente bloqueador $\mathrm{H}-2$ (ranitidina $300 \mathrm{mg} /$ día por 4 semanas); antibiótico (amoxicilina 1,5-2,0 g/día por 1 semana), y antiparasitario (metronidazol $750 \mathrm{mg}$ /día por 1 semana); y el otro esquema quimioterapéutico terapia triple novedosa, que utiliza un inhibidor de la bomba de protones (omeprazol $40 \mathrm{mg}$ /día por 4 semanas), en asociación con antibióticos de amplio espectro de acción (claritromicina $500 \mathrm{mg} /$ día y amoxicilina $1,5 \mathrm{mg} /$ día) por una semana de tratamiento (Collazo, Haedo, 1998).

La investigación se realizó en el Instituto Nacional de Gastroenterología de Cuba, donde se ha comprobado la efectividad terapéutica de los tratamientos antiulcerosos, mediante la realización de un estudio clínico prospectivo en dos grupos de 127 pacientes para cada uno de estos esquemas quimioterapéuticos.

La efectividad terapéutica se mide por la acción que tienen los fármacos para posibilitar la cicatrización de las úlceras pépticas y la erradicación de agente causante $(H$. pylori), y el indicador utilizado para medir la efectividad del tratamiento es el caso curado de esta enfermedad. (Mc Coy, Blaney-Chandramouli, Mutnick, 1998; Fernández et al., 1998).

En nuestro país, los parámetros para evaluar los resultados de la efectividad del tratamiento son los siguientes:

- Realizar una endoscopía diagnóstica para determinar la presencia de la úlcera en los tejidos y si el resultado es positivo, se comienza con el esquema de tratamiento indicado.

- Después de terminado el tratamiento, se realiza una endoscopía comprobatoria para valorar la evolución en la cicatrización de la úlcera. Si esta no es favorable, se procede a realizar una biopsia para analizar muestra de los tejidos y la permanencia del $H$. pylori en la misma.

- Inmediatamente después se prosigue un nuevo ciclo de tratamiento con otro esquema farmacológico, y una vez finalizada la farmacoterapia empleada, se efectúa otra endoscopía comprobatoria para observar si ha podido cicatrizar la lesión ulcerosa.

Para la aplicación de estos esquemas de tratamientos se utilizaron fármacos importados, algunos de ellos son especialidades farmacéuticas y otros son genéricos. Los costos unitarios de adquisición por unidad de presentación (comprimidos o cápsulas) de estos medicamentos/esquemas de tratamiento, tienen el importe siguiente:

Terapia Triple Convencional compuesta por el esquema Ranitidina:

Esquema Ranitidina: ranitidina $150 \mathrm{mg}(\$ 1,20$ / comprimido), amoxicilina $250 \mathrm{mg}(\$ 0,80 /$ cápsula), y metronidazol $250 \mathrm{mg}$ (\$0,25 / comprimido).

Terapia Triple Novedosa integrada por el esquema del Omeprazol:

Esquema Omeprazol: omeprazol $20 \mathrm{mg}(\$ 1,94$ / cápsula), claritromicina $250 \mathrm{mg}$ ( $\$ 1,14$ / comprimido), y amoxacilina $250 \mathrm{mg}$ ( $\$ 0,80$ / cápsula). 
Los costos de los tratamientos/esquemas se estiman sobre la base de la multiplicación del costo unitario del medicamento analizado por unidad de presentación, por la dosis diaria indicada para su uso, y por el ciclo completo de tiempo de su administración por paciente (Bootman, Towsend, McGhan, 1991). Estos importes de los tratamientos se comparan entre sí, para calcular la diferencia de los gastos que ofrece una alternativa farmacológica con relación a la otra, durante el tiempo de tratamiento de cuatro semanas de duración.

La evaluación económica de los tratamientos se realizará mediante la técnica del Análisis Costo-Efectividad (ACE) para las diferentes alternativas farmacológicas y de esta forma obtener la eficiencia del tratamiento, que no es más que la relación existente entre los costos de la farmacoterapia y la efectividad terapéutica alcanzada (Sacristán del Castillo et al., 1994). El ACE puede establecerse mediante dos tipos de análisis, la relación costo-efectividad medio (ACEM), que establece una comparación entre el costo por unidad de efectividad de las dos opciones y el análisis costo-efectividad incremental (ACEI) e que se obtiene dividiendo el incremento de los costos por el de los efectos sobre la salud de las alternativas analizadas, y se expresa como el costo de una opción por unidad de efectividad adicional respecto a la otra (Antoñanzas Villar, 1995).

Se realiza el análisis de decisión farmacoeconómica para comparar las razones o indicadores que resumen los resultados de cada opción (Antoñanzas Villar, 1995), empleando el árbol de decisión, que es un diagrama que representa el conjunto de posibles sucesos o cursos de acción que puede ocurrir como resultado de una decisión (SOIKOS, 1996), en las condiciones de la práctica clínica habitual.

\section{Resultados}

En el esquema quimioterapéutico que utiliza la ranitidina, la efectividad promedio es de un $83 \%$ de los casos curados, y para la alternativa en que se emplea el omeprazol, se considera que tiene un $95 \%$ de efectividad de los casos tratados. Todos los pacientes que participaron en ambos grupos de estudios, completaron el tiempo de tratamiento por espacio de 4 semanas, sin haber ocurrido abandonos voluntarios, ó tener que interrumpir la administración de la farmacoterapia debido a la aparición de reacciones adversas de significativa importancia, que haya requerido de un tratamiento secundario para las mismas. El tiempo total de observación en los pacientes, una vez finalizado el tratamiento antiulceroso fue de 3 meses.

Con respecto al costo total de la farmacoterapia empleada, el menor importe del tratamiento lo tiene el esquema farmacológico que emplea la alternativa de la ranitidina con $\$ 12348.21$ para los 127 pacientes tratados (costo promedio de \$97,23/paciente); y el tratamiento más costoso lo tiene el esquema del omeprazol con $\$ 17931.13$ para el total de los casos con tratamiento (costo promedio de \$141,19/paciente).

La evaluación farmacoeconómica se centrará en la relación costo-efectividad de estas alternativas del tratamiento. El ACEM del esquema de la ranitidina es de \$ $117,14 /$ caso curado, mientras que la opción que utiliza el omeprazol es de $\$ 148,62 /$ caso curado; siendo la diferencia comparativa entre ambas alternativas de $\$ 31,48$ /caso curado a favor del esquema quimioterapéutico que utiliza la ranitidina.

Pero como la alternativa farmacológica del omeprazol tiene una mayor efectividad terapéutica que el esquema de la ranitidina, se tiene que recurrir al ACEI de esta opción en comparación con la ranitidina, arrojando un resultado no satisfactorio de $\$ 348.93$ /caso curado adicional, aspecto este que incide negativamente en los niveles de eficiencia de este tratamiento, y lo limita en su uso generalizado para toda la población afectada por esta enfermedad en nuestro país.

Como se puede apreciar en las Tablas I y II, aunque los dos esquemas farmacológicos (ranitidina y omeprazol) tienen niveles diferentes de efectividad, se puede considerar que ambas terapias alcanzan resultados satisfactorios en la curación de las úlceras, siendo la mayor diferencia-

TABLA I - Análisis Costo-Efectividad Medio de los Tratamientos Antiulcerosos

\begin{tabular}{lccc}
\hline Indicadores & $\begin{array}{c}\text { Alternativa 1 } \\
\text { Esquema Ranitidina }\end{array}$ & $\begin{array}{c}\text { Alternativa 2 } \\
\text { Esquema Omeprazol }\end{array}$ & Diferencia \\
\hline Costo Prom. Tratamiento(\$/paciente tratado) & 97.23 & 141.19 & 43.96 \\
Efectividad Terapéutica(\% de casos curados) & 83.0 & 95.0 & 12.0 \\
Número de casos curados & 105 & 121 & 16 \\
Costo-Efectividad Medio(\$caso curado) & 117.14 & 148.62 & 31.48 \\
\hline
\end{tabular}

$(\mathrm{n}=127$ pacientes tratados)

Fuentes: Instituto Nacional de Gastroenterología (Cuba), Centro de Investigación y Desarrollo de Medicamentos (Cuba) 
TABLA II - Costo-Efectividad Incremental (ACEI) de los Tratamientos Antiulcerosos.

$\mathrm{ACEI}=\frac{\mathrm{C}_{2}-\mathrm{C}_{1}}{\mathrm{E}_{2}-\mathrm{E}_{1}}=\frac{\mathrm{DC}}{\mathrm{DE}}$

Donde:

$\mathrm{C}_{2}($ Importe del Costo Esquema Omeprazol $)=\$ 17931.13 ; \mathrm{C}_{1}$ (Importe del Costo Esquema Ranitidina) $=\$ 12348.21$ $\mathrm{E}_{2}($ Efectividad Esquema Omeprazol $)=121$ casos curados; $E_{1}($ Efectividad Esquema Ranitidina $)=105$ casos curados

$\mathrm{ACEI}=\frac{17931.13-12348.21}{121-105}=\frac{5582.92}{16}$

\section{Comparación de Alternativas de Tratamientos}

$\begin{array}{cc}\text { Diferencia } & \text { Diferencia } \\ \text { Incremento de } & \text { Incremento de } \\ \text { Costos } & \text { Efectividad } \\ (\$) & \text { (casos curados) }\end{array}$

16

\section{Costo-Efectividad \\ Incremental \\ (\$/caso curado \\ adicional)}

348.93

Esquema Omeprazol vs Esquema Ranitidina 5582.92

$(\mathrm{n}=127$ pacientes tratados $)$

Fuentes: Instituto Nacional de Gastroenterología, (Cuba); Centro de Investigación y Desarrollo de Medicamentos (Cuba)

ción existente entre las mismas, los costos incurridos y los niveles de eficiencia alcanzados con el tratamiento. Ante esta situación, se realiza el análisis de decisión farmacoeconómica para comparar los resultados de cada opción de tratamiento, y se representa mediante el empleo del árbol de decisión, cuyo resultado es el siguiente:

Con el empleo del análisis farmacoeconómico, se obtiene una estrategia farmacoterapéutica compuesta por la combinación en dos ciclos sucesivos de los tratamientos farmacológicos ranitidina-omeprazol, que incrementará la efectividad clínica en un 99\% de los casos curados, y tendrá un ACEM de \$122.71/caso curado para el curso del seguimiento de esta enfermedad.

\section{Discusion}

El uso clínico de los bloqueadores H-2, en este caso la ranitidina, se centra en su capacidad para inhibir la secreción del ácido gástrico en estados hipersecretorios, particularmente aquellos que involucran una úlcera péptica (Lasser, Burlage, Fulsch, 1994; Mc Coy, BlaneyChandramouli, Mutnick, 1998); y el omeprazol, prototipo de los inhibidores de la bomba de protones que bloquea la secreción gástrica ácida por inhibición (Wash, 2000) Los antibióticos se utilizan para combatir la relación entre la úlcera péptica y el $H$. pylori, y particularmente, los efectos beneficiosos por la erradicación de este microorganismo (Penston, 1994; Fernández et al., 1998).

En varios trabajos, se ha demostrado la evidencia clínica del uso de la ranitidina combinada con amoxicilina y metronidazol para la erradicación del H. pylori en

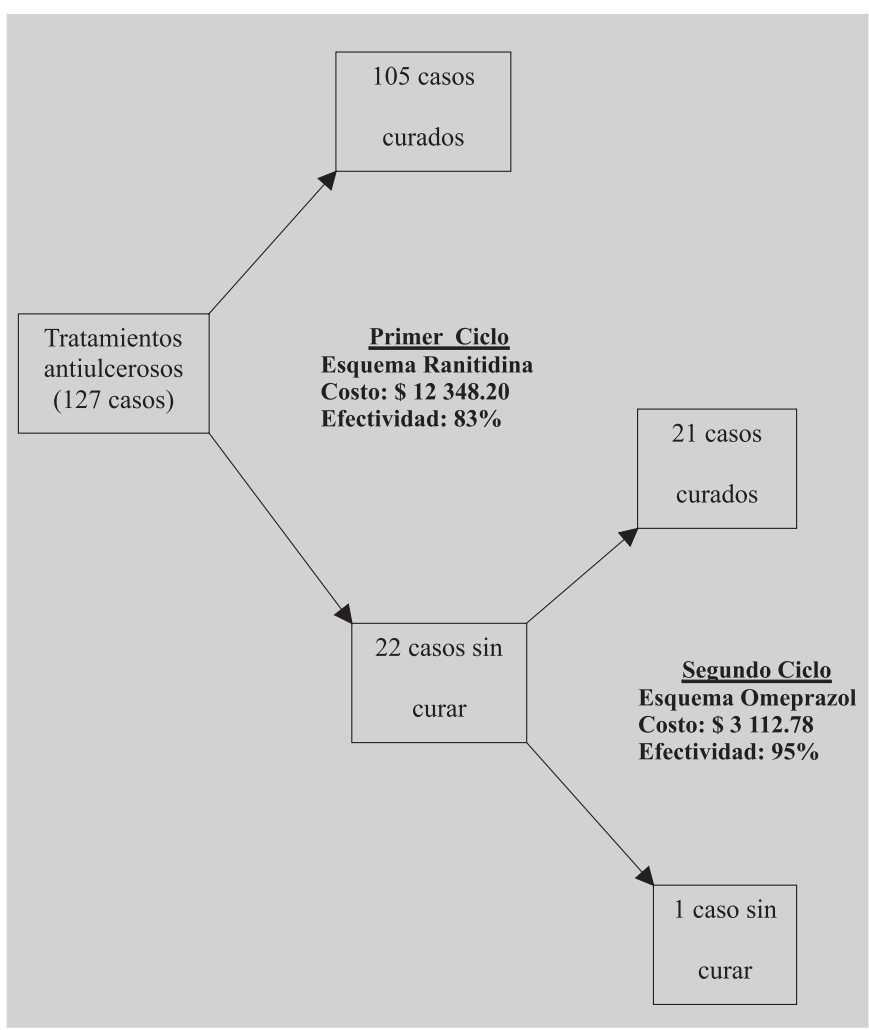

FIGURA 1 - Análisis de Decisión Farmacoeconómica. Arbol de Decisión.

aproximadamente el $90 \%$ de los pacientes. Esta terapia combinada reduce la recurrencia de úlcera duodenal significativamente (Fernández et al., 1998; Vademecum, Glaxo SmithKline, 2001). 
TABLA III - Resultado Final. Estrategia del Tratamiento Combinado Ranitidina - Omeprazol

\begin{tabular}{|c|c|c|c|c|}
\hline \multirow{2}{*}{$\begin{array}{l}\text { Esquemas de } \\
\text { Tratamientos }\end{array}$} & \multirow{2}{*}{$\begin{array}{c}\text { Importe Costo de } \\
\text { Tratamiento (\$) }\end{array}$} & \multicolumn{2}{|c|}{ Resultados Obtenidos } & \multirow{2}{*}{$\begin{array}{c}\text { Efectividad } \\
(\% \text { casos curados })\end{array}$} \\
\hline & & Casos Curados & Casos sin Curar & \\
\hline Primer Ciclo-Ranitidina & 12348.20 & 105 & 22 & 83 \\
\hline Segundo Ciclo-Omeprazol & 3112.78 & 21 & 1 & 95 \\
\hline Tto. Combinado & 15460.98 & 126 & 1 & 99 \\
\hline Ranitidina - Omeprazol & & & & \\
\hline
\end{tabular}

$(\mathrm{n}=127$ pacientes tratados)

Costo Efectividad Medio: $\$ 15$ 460.98/126 = \$122.71/caso curado (Eficiencia Total del Tratamiento)

Fuente: Instituto Nacional de Gastroenterología (Cuba); Centro de Investigación y Desarrollo de Medicamentos (Cuba)

También se ha podido comprobar en la practica clínica que el tratamiento para las úlceras con el omeprazol es efectivo (Levy et al., 2001), pero es más costoso que la utilización de un esquema farmacoterapéutico con la ranitidina, cuestión esta que puede conspirar en contra de su eficiencia en el tratamiento (Schupp, Schrand, Mutnick, 2003)

Es por esta razón, que se plantea el establecimiento de una estrategia farmacoterapéutica compuesta por la combinación sucesiva en dos ciclos de los tratamientos, ya que se pudo comprobar en la práctica clínica que ambos esquemas son efectivos en el tratamiento. De esta forma, las pautas a seguir para conformar la estrategia del tratamiento son las siguientes:

- Primero, un ciclo de tratamiento con el esquema quimioterapéutico que utiliza la ranitidina para su uso generalizado de los pacientes en el Sistema Nacional de Atención en Salud.

- Posteriormente, para los casos específicos que no resuelven con la alternativa de la ranitidina, un segundo ciclo de tratamiento con el empleo del esquema con el omeprazol.

\section{EVALUACIÓN ECONÓMICA DEL USO DE CEFAZOLINA VERSUS CEFTRIAXONA EN LA PROFILAXIS PERI-OPERATORIA}

El otro estudio realizado trata sobre la utilización de las cefalosporinas parenterales por vía intravascular (específicamente intravenosa), de la cefazolina en comparación con la ceftriaxona, para la profilaxis peri-operatoria de distintas intervenciones quirúrgicas, como son: apendicitis agudas, histerectomías y cirugía de colon.

\section{Materiales Y Metodos}

Las cefalosporinas son antibióticos muy usados. Son efectivos para el tratamiento de infecciones del tracto respiratorio, de la piel y los tejidos blandos, huesos y articulaciones, tracto urinario y torrente circulatorio. Este grupo de medicamentos se usa ampliamente en cirugía para la profilaxis de la infección (Travieso, Carnot, Castro, 1990; Hardman, Limbird, Gilmann, 1996; Rosenstein, 2001).

La cefazolina es un representante de este grupo perteneciente a la primera generación con un amplio espectro de aplicación y está indicado en el tratamiento de un extenso conjunto de infecciones y en la profilaxis quirúrgica (Parfitt, 1999; Catalogo de Especialidades Farmacéuticas, España, 2000). La ceftriaxona es una de las cefalosporinas conocidas que tiene un mayor tiempo de vida útil y este aspecto permite la utilización de una dosis diaria en la administración del antibiótico parenteral (Schwicker, Dinkel, 1991).

La evaluación clínica se realizó durante un año en el Hospital Clínico Quirúrgico "Joaquín Albarrán” en la Ciudad de La Habana, a un total de 104 casos con cefazolina y 107 pacientes con ceftriaxona. De esta forma, se desarrolló un estudio prospectivo basado en el criterio de monoterapia profiláctica perioperatoria con ambos antibióticos.

Con la cefazolina se atendieron 39 casos de apendicitis aguda (1dosis de $1 \mathrm{~g} /$ paciente), 35 casos de histerectomía ( 2 dosis de $1 \mathrm{~g} /$ paciente); y 30 casos de cirugía de colon ( 3 dosis de $1 \mathrm{~g} /$ paciente). Para la profilaxis recibieron ceftriaxona 39 casos de apendicitis aguda (1 dosis de $1 \mathrm{~g} /$ paciente), 33 casos de histerectomía (1 dosis de $1 \mathrm{~g} /$ paciente), y 35 casos de cirugía de colon (1 dosis de $1 \mathrm{~g} /$ paciente) (Moya et al., 2001).

La efectividad terapéutica de la profilaxis con las cefalosporinas utilizadas se mide por la prevención de sepsis post-operatorias en los pacientes intervenidos quirúrgicamente, y se expresan en el indicador por ciento de casos con infecciones evitadas (Moya et al., 2001).

Los parámetros para evaluar los resultados de la efectividad en la profilaxis desde el punto de vista de la infección, son los siguientes: 
- No presencia de cuadro febril en el paciente intervenido quirúrgicamente.

- Ausencia de aparición de los signos de inflamación local en la herida.

- Comprobación de que el leucograma realizado se mantenga dentro de los límites normales.

Para realizar la quimioprofilaxis quirúrgica se utilizaron medicamentos genéricos de importación. Los costos unitarios de adquisición de estos fármacos son los siguientes: cefazolina $1 \mathrm{~g}$ inyectable es de $\$ 2,28 /$ bulbo, y para la ceftriaxona $1 \mathrm{~g}$ inyectable es de $\$ 7,00 /$ bulbo. Los costos de los tratamientos se calculan sobre la base de la multiplicación del costo unitario del medicamento seleccionado por la dosis diaria de su administración/paciente.

Con estos efectos sobre la salud (efectividad) y sobre los recursos (costos), la evaluación económica se realiza mediante el ACE de las alternativas analizadas. El ACE se obtiene mediante la relación existente entre los costos de los tratamientos farmacológicos y él por ciento de efectividad terapéutica alcanzado con esa alternativa de trata- miento (Collazo, Flores, 1999). De esta forma, se obtienen la eficiencia promedio ( $\$$ / infección evitada) y en términos incrementales (\$/ infección evitada adicional) de la profilaxis para las distintas intervenciones quirúrgicas

\section{Resultados}

Los resultados alcanzados de la efectividad terapéutica para las distintas alternativas de profilaxis peri-operatorias con las cefalosporinas, se exponen en la Tabla IV.

El costo de la quimioprofilaxis con cefazolina en la apendicitis aguda es de $\$ 2,28$ / paciente, en la histerectomía es de $\$ 4,56 /$ paciente, y para la cirugía de colon es de $\$$ $6,84 /$ paciente. Para la ceftriaxona, el costo de la profilaxis peri-operatoria tiene el mismo importe de 7,00/paciente para todas las intervenciones quirúrgicas analizadas.

Los resultados de la eficiencia promedio y en términos incrementales de la administración parenteral para la profilaxis de las infecciones post-operatorias en las distintas intervenciones quirúrgicas, se expresan en las Tablas V y VI.

TABLA IV - Efectividad de la Cefazolina y la Ceftriaxona en la Profilaxis Peri-operatoria

\begin{tabular}{lcc}
\hline Intervención Quirúrgica & Cefazolina(\% infecciones evitadas) & Ceftriaxona(\% infecciones evitadas) \\
\hline Apendicitis Aguda & 89,7 & 92,3 \\
Histerectomía & 94,3 & 90,9 \\
Cirugía de Colon & 90,0 & 91,4 \\
\hline
\end{tabular}

Fuente: Hospital Clínico Quirúrgico “Joaquín Albarrán”(Cuba)

TABLA V - Eficiencia Promedio de la Cefazolina y Ceftriaxona en la Profilaxis Peri-operatoria

\begin{tabular}{lcc}
\hline Intervención Quirúrgica & Cefazolina(\$infección evitada) & Ceftriaxona(\$infección evitada) \\
\hline Apendicitis Aguda & 2,54 & 7,58 \\
Histerectomía & 4,84 & 7,70 \\
Cirugía de Colon & 7,60 & 7,65 \\
\hline
\end{tabular}

Fuente: Centro de Investigación y Desarrollo de Medicamentos (Cuba)

TABLA VI - Análisis Costo-Efectividad Incremental para la Profilaxis Peri-operatorias

\begin{tabular}{lccc}
\hline $\begin{array}{l}\text { Comparación de } \\
\text { Ceftriaxona vs. Cefazolina }\end{array}$ & $\begin{array}{c}\text { Diferencia } \\
\text { Incremento de } \\
\text { Costos } \\
\text { (\$/paciente) }\end{array}$ & $\begin{array}{c}\text { Diferencia } \\
\text { Incremento de } \\
\text { Efectividad } \\
\text { (infección evitada) }\end{array}$ & $\begin{array}{c}\text { Costo-Efectividad } \\
\text { Incremental } \\
\text { (\$/infección evitada } \\
\text { adicional) }\end{array}$ \\
\hline Apendicitis Aguda & 4.72 & 0.026 & 181.54 \\
Histerectomía & 2.44 & $(0.031)$ & No procede \\
Cirugía de Colon & 0.16 & 0.014 & 11.43 \\
\hline
\end{tabular}

Fuente: Centro de Investigación y Desarrollo de Medicamentos(Cuba) 
Con este resultado, se puede establecer una política para el uso de estos antibióticos en el hospital. Para los casos de las intervenciones quirúrgicas de apendicitis agudas e histerectomías, la alternativa farmacológica más favorable desde el punto de vista de la eficiencia (primera opción de la quimioprofilaxis) es la cefazolina, tanto por el ACEM como en términos incrementales. Para la cirugía de colon, ambas alternativas de prevención de sepsis post-quirúrgicas tienen resultados costo-efectividad muy similares; pero desde el punto de vista de su administración, la ceftriaxona supera a la cefazolina por tener una sola dosis parenteral/paciente, mientras que la cefazolina se debe administrarse en tres dosis durante la intervención quirúrgica/paciente. Por lo tanto, la ceftriaxona es la primera opción farmacológica como esquema general para la profilaxis de infecciones post-operatorias en casos de cirugía de colon.

\section{Discusion}

El tratamiento con antibacterianos resulta una de las terapias de mayor costo en los pacientes hospitalizados; siendo el aspecto más importante la curación de estos; pero siempre debe tenerse en cuenta que el antibiótico más costoso no es el mejor de forma individual; si no que debe hacerse un análisis que permita obtener el mayor efecto sobre la salud a un costo razonable (Shulkin, 1993). Es por ello, que cada vez es mayor el interés por el análisis y control de los costos de la farmacoterapia hospitalaria, y por la determinación de estrategias para su contención (Zweifel, 1988).

Con respecto a la cefazolina, aunque es una cefalosporina de primera generación y no tiene reportado un espectro de acción tan amplio como lo tiene la ceftriaxona, pero en la práctica clínica real en nuestro país ha demostrado ser más eficiente de forma general, para la prevención de las infecciones post-quirúrgicas que la ceftriaxona, debido fundamentalmente a su bajo importe del costo en la quimioprofilaxis de las sepsis post-operatorias.

La ceftriaxona tiene como ventaja la administración de una dosis única en el acto quirúrgico para la prevención de infecciones post-operatorias. Este último elemento repercute favorablemente en el costo total de la farmacoterapia empleada en comparación con otras opciones alternativas. En este sentido, varios autores han reportado diversos aspectos del impacto socioeconómico y de los costos potenciales ahorrados con el uso de una dosis diaria de tratamiento con ceftriaxona (Travieso, Carnot, Castro, 1990; Schwicker, Dinkel, 1991).

También es de resaltar en el caso de ceftriaxona, los problemas que puede tener su uso en la profilaxis quirúrgica, ya que una utilización irracional de este fármaco puede generar la inducción de resistencias en el ámbito hospitalario. Es por esta razón, que su administración no debe ser de forma generalizada en la prevención de infecciones post-quirúrgicas, y solamente deberá emplearse en casos de cirugía bien argumentados. En Cuba, la utilización de esta cefalosporina de tercera generación está bien controlada, y se emplea fundamentalmente en los casos de profilaxis de cirugías de colon y rectal, y excepcionalmente, cuando el cuadro clínico y quirúrgico del paciente aconsejan que es el antibiótico de elección.

Con la implementación del criterio farmacoeconómico, se podrá establecer una estrategia de racionalización farmacoterapéutica intrahospitalaria; así como también incrementar la eficiencia en la administración de las cefalosporinas para la profilaxis de infecciones post-quirúrgicas.

\section{CONCLUSIONES}

- Los estudios farmacoeconómicos realizados en Cuba, han demostrado la aplicación práctica de esta útil herramienta de trabajo en la toma de decisiones sanitarias, y poder elaborar de esta manera, las políticas y estrategias para la utilización de los medicamentos, sobre la base de su efectividad clínica comprobada y los costos de la farmacoterapéutica empleada.

- La implementación del criterio farmacoeconómico en los tratamientos farmacológicos antiulcerosos, permite establecer una estrategia farmacoterapéutica compuesta por la combinación en dos ciclos sucesivos de tratamientos con ranitidina y omeprazol, que aumente la efectividad terapéutica e incremente la eficiencia del tratamiento para el curso del seguimiento de esta enfermedad.

- La farmacoeconomía aplicada a la utilización de los antibióticos en el hospital, permite establecer una política para su uso racional y eficiente, específicamente en lo referente al empleo de las cefalosporinas para la profilaxis peri-operatoria de intervenciones quirúrgicas, al poder evaluar desde el punto de vista técnico y económico, las opciones para la administración de cefazolina en comparación con la ceftriaxona.

\section{ABSTRACT}

\section{The practical application of Pharmacoeconomics in the use of drugs in Cuba}

The purpose of this work is to expose how pharmacoeconomic studies contribute to render a rational and efficient use to drugs. As a consequence of the present study focusing on pharmacoeconomics by the Cuban-Republic sanitary authorities, the 
pharmaceutical industry is becoming interested in such analytical techniques and has started to use them for developing its strategies for research, development, production and marketing of its products. The practical application of these analysis techniques to the sanitary field represents one of most important initial efforts made in Cuba, which is guided to attain that the pharmacoeconomic evaluation starts to be involved in the process of rational use of drugs. Through practical examples of pharmacoeconomic studies jointly performed between the pharmaceutical sector and that one of medical care in our country, different investigations regarding to antiulcer pharmacological treatments and perioperatory antimicrobial prophylaxis are exponed. Their application were presented in taking sanitary decisions based on demonstrated clinical efficiency and costs of pharmaceutical therapy used.

UNITERMS: Pharmacoeconomics. Cost-effectiveness. Antiulcer treaments. Post-operatory prophylaxis. Rational use.

\section{REFERENCIAS BIBLIOGRÁFICAS}

ANDERSON, F. Why is the pharmaceutical industry investing increasing amounnts in heath economics evaluations? Int. J. Tech. Assess. Health Care, v.11, p. 750-761, 1995.

ANTOÑANZAS VILLAR, F. Evaluación económica aplicada a los medicamentos: características y metodología. In: SACRISTAN DEL CASTILLO, J.A.; BADIA LLACH, X.; ROVIRA FORNS, J., eds. Farmacoeconomía: Evaluación económica de medicamentos. Madrid: Editores Médicos, 1995. cap. 2, p. $31-50$.

BADIA, X.; ROVIRA, J. Evaluación económica de medicamentos. Un instrumento para la toma de decisión práctica clínica y política sanitaria. Barcelona: Luzán 5, 1994. p. 19.

BOOTMAN, J.L.; TOWSEND,R.J.; Mc GHAN, W.F. Principles of pharmacoeconomics. Cincinnati: Harvey Whitney Book Company, 1991. cap. 1, p. 84-97.

\section{CANADIAN COORDINATING OFFICE FOR HEALTH}

TECHNOLOGY. Guidelines for economic evaluation of pharmaceutics: Canada. 1. ed. Ottawa: CCOHT, 1994. p. 1.
COllazO, M.; CASADEMUNT, N. La farmacoeconomía en la industria farmacéutica y el sistema sanitario de Cuba. Rev. Panam. Salud Pública, v.10, n.4, p. 263-267, 2001.

COLLAZO, M.; FLORES, N. Farmacoeconomía. La evaluación de la eficiencia de los tratamientos farmacológicos. Rev. Cubana Farm., v.34, n. 1, p. 63-69, 1999.

COLLAZO, M.; HAEDO, W. Evaluación económica comparativa de los tratamientos quimioterapéuticos antiulcerosos. Rev. OFIL; v.9, n.1, p. 20-26, 1998.

CONSEJO GENERAL DE COLEGIOS OFICIALES DE FARMACEUTICOS. Catalogo de especialidades farmacéuticas. Madrid: Ediciones Informatizadas, 2000. p. $1175-1176$.

DRUMMOND M.F.; STODDART, G.L.; TORRANCE, G.W. Methods for the economic evaluation of health care programmes. 1. ed. Toronto: Oxford Medical Publications, 1987.p. 19-38.

EVANS, D. The changing healthcare environment. PharmacoEconomics, v.1, n. 1, p.5-7, 1992.

FERNANDEZ, J.; LOPEZ DE ANDRES, A.; ZAPATER, P.;ABAD, F. Análisis coste-efectividad de la erradicación del Helicobacter pylori como tratamiento de la úlcera duodenal. An. Med. Interna. v.15, n.10, p. 515-522, 1998.

GLAXO SMITHKLINE. Vademecum de productos. 1. ed. Ciudad de México: Ediciones PLM, 2001. p. 174-176.

GLENNIE, J. Pharmacoeconomics. What every pharmacist should know. Pharm. Connect., v. Jan/Feb, p. 8-15, 1995.

HARDMAN, J.; LIMBIRD, L.; GOODMAN GILMAN, A. Las bases farmacológicas de la terapéutica. 9. ed. Ciudad de México: Mc Graw-Hill Interamericana Editores, 1996. v.2, p. 1158-1166.

LASSER, C.; BURLAGE, M.; FULSCH, U. Random comparative studies with crosslinking interchange in ranitidine effervecesnt tablets (300mg) vs famotidine coating tablets (400 mg) Drug Res., v.3, n.5, p. 626-29, 1994. 
LEVY, M.J.; SEELIG, C.B.; ROBINSON, N.J.; RANNEY, J.E. Comparison of omeprazole and ranitidine for stress ulcer prophylaxis. Dig. Dis. Sci., v.42, p. 1255-1259, 2001.

Mc COY, S.; BLAYNEY-CHANDRAMOULIi, J.; MUTNICK, A. Using multiple pharmacoeconomic methods to conduct a cost-effectiveness analysis of histamine $\mathrm{H}_{2}$-receptor antagonists. Am. J. Health Syst. Pharm., v. 55, suppl. 4, p. S8-12, 1998.

MOYA, A., COLLAZO, M., PISONERO, J., PARDO, G. Evaluación económica del uso de cefazolina versus ceftriaxona en la profilaxis perioperatoria. Rev. Cubana Farm., v. 34, n.3, p. 187-91, 2001

PANERAI, R., MOHR, J. Evaluación de tecnologías en salud. Metodología para países en desarrollo. Washington D.C: Organización Panamericana de la Salud, 1990. p. 925.

PARFITT, K. Martindale. The complete drug reference. 32. ed. London: Pharmaceutical Press, 1999. p.181.

PENSTON, J.G. Helicobacter pylori erradication-under standable caution but no excuse for inerti. Alim. Pharm., v. 3, n.1, p. 369-89, 1994.

ROSENSTEIN, E. Diccionario de especialidades farmacéuticas. 47. ed. Ciudad de México: Editorial PLM, 2001. p. 223-26.

SACRISTAN DEL CASTILLO, J.A. Farmacoeconomía y evaluación de medicamentos. In: SACRISTAN DEL CASTILLO, J.A.; BADIA LLACH, X.; ROVIRA FORNS, J., eds. Farmacoeconomía: Evaluación económica de medicamentos. Madrid: Editores Médicos, 1995. cap. 1, p. 19-30.

SACRISTAN, J.A.; SOTO, J.; REVIRIEGO, J.; GALENDE, I. Farmacoeconomía: el cálculo de la eficiencia. Med. Clin., v.103, p. 143-149, 1994.
SCHUPP, K.; SCHRAND, L.; MUTNICK, A.A costeffectiveness analysis of stress ulcer prophylaxis. Ann. Pharmacother., v.37, p. 631-635, 2003.

SCHWICKER, D.; DINKEL, R. The socioeconomic evaluation of ceftriaxone. An international review of the socioeconomic aspects of once-daily ceftriaxone antibiotic treatment. Basel: Health Econ, 1991. p 2.

SHULKIN, D. The rising cost of pharmaceuticals: a physician perspective. Am. J. Hosp. Pharm., v. 50, supl. 4, p. 8-10, 1993.

SOIKOS. Glosario de términos y conceptos de uso frecuente en la evaluación económica de medicamentos y programas sanitarios. Barcelona: Química Farmacéutica Bayer, 1996. p. 11.

SOTO, J. Evaluación económica de medicamentos: perspectiva de la industria farmacéutica en nuestro país. Rev. Atenc. Farm., v.1, n.6, p. 600-608, 1999.

TRAVIESO, M.; CARNOT, J.; CASTRO, A. Uso de cefalosporinas a nivel hospitalario. Em Pauta- Acta Med. Antibiot. Hosp. Clín. Quir. Hermanos Ameijeiras. La Habana, v. 4, n. 2, p. 224-237, 1990.

TRILLO, J.L.; AZNAR, J. Bases de la evaluación económica de medicamentos. Rev. Atenc. Farm., v.1, p. 5, p. 469-56, 1999.

VELAZQUEZ, G. Farmacoeconomía: evaluación científica o estrategia comercial? Rev. Panam. Salud Pública, v.5, n. 1, p. $54-7,1999$.

WALSH, P. Physicians desk reference. PDR. 54. ed. New Jersey: Medical Economics Company, 2000. p. 617-621.

ZWEIFEL, P. Socioeconomic evaluation of drugs therapy. Berlin: Spinger Verlag, 1988, p. 34-41.

Recebido para publicação em 18 de setembro de 2003. Aceito para publicação em 21 de outubro de 2004. 